\title{
Contribution à l'analyse microbiologique des poulets, des chinchards (Trachurus trachurus) et des poissons salés vendus à Kinshasa en vue de la sensibilisation à la méthode ISO- 22000 :2005 HACCP
}

Espérance Bumona Zayukua ${ }^{1}$, Joachim di M’balu Umba ${ }^{2}$, Charles Nzau Kusika $^{3}$, Thaddée Ndyanabo Masimango ${ }^{4}$, Jean Grâce Ndongala Lufimpadio ${ }^{5}$.

1. Institut Supérieur AgroVétérinaire Saint Pierre Canisius de Kimwenza, B.P. 3724 Kinshasa-Gombe

2. Faculté de Médecine Vétérinaire, Dpt de Cliniques, Université Pédagogique Nationale, B.P. 8815 Kinshasa-Ngaliema

3. Institut Supérieur des Techniques Appliquées en Chimie Agro Alimentaire de Kimpese/ Kongo Central

4. Faculté des Sciences Agronomiques, Dpt de Chimie et Industries Agricoles, Université de Kinshasa, B.P. 190 Kinshasa 11

5. Faculté des Sciences, Dpt de Chimie, Université Pédagogique Nationale, B.P. 8815 Kinshasa-Ngaliema

Email Corresponding : joachimumba@yahoo.fr

Mots clés : Poulets, Chinchards, Poisons salés, Contamination, Sécurité sanitaire, ISO- 22000 : 2015

Publication date 30/11/2019, http://www.m.elewa.org/JAPS

\section{RESUME}

La qualité des denrées alimentaires est devenue une préoccupation majeure pour les consommateurs des nombreux pays. En effet, ces dernières décennies, le monde a connu des cas d'encéphalopathie spongiforme bovine (ESB) au Royaume-Uni et d'autres problèmes alimentaires plus communs associés à la Salmonella, à l'Escherichia Coli enterohaemorrhagique, de tremblante du mouton, du poulet à la dioxine, de Listériose . Cette situation a amené les consommateurs avertis à exiger que les aliments qui leurs sont vendus, soient sans danger. Les surgelés comptent parmi les aliments les plus consommés dans les grandes villes de la RD Congo. Cependant, une bonne partie de ces produits subit une rupture de la chaîne de froid telle qu'elle se détériore et devient nuisible à la santé des consommateurs. Nous savons que le poisson est une denrée très périssable et nécessite le maintien en continu de la chaîne de froid. Or, les coupures de fournitures d'énergies sont récurrentes dans notre capitale. Et cela devient un dilemme pour les consommateurs kinois. Quel est l'impact de la rupture de la chaîne de froid sur la qualité de ces surgelés vendus sur les marchés de Kinshasa? Comment le consommateur kinois est-il protégé contre les dangers alimentaires de la fourche à la fourchette?

L'objectif de la présente recherche basée sur les techniques d'isolements, d'identification et de dénombrement et conformément aux normes ISO spécifiques à chaque germe, est de faire une analyse microbiologique du poulet emballé, déballé et découpé, du poisson chinchard sorti du carton, exposé sur les étals et du poisson salé local, importé et poisson du fleuve Congo vendus sur quelques marchés de Kinshasa, étudier les enjeux de la sécurité sanitaire par la méthode ISO-22000:2005 - HACCP et proposer à l'autorité publique l'application de ces principes d'assainissement, de les vulgariser et renforcer les mesures d'hygiène alimentaire dans les grandes agglomérations du pays au profit de la santé publique (accumulation de déchets dans les rues et congestion des égouts) et lutter contre les toxi-infections alimentaires collectives (TIAC). 
Il ressort de notre analyse que la fréquence de contamination de micro-organismes, les Proteus arrivent en tête avec 63\%, suivis des Klebsiella $41 \%$, les Escherichia coli 21\%, les Citrobacters et Enterobacters $20 \%$ et Salmonella spp.et Shigella 8\%.

\section{ABSTRACT}

Food quality has become a major concern for consumers in many countries. Indeed, in recent decades, the world has experienced cases of bovine spongiform encephalopathy (BSE) in the United Kingdom and other more common nutritional problems associated with Salmonella, enterohaemorrhagic Escherichia coli, scrapie, chicken with dioxin, listeriosis. This situation has led savvy consumers to demand that the food sold to them be safe. Frozen foods are among the most consumed foods in the big cities of DR Congo. However, a good part of these products undergo a break in the cold chain as it deteriorates and becomes harmful to the health of consumers. We know that fish is a very perishable commodity and requires the continuous maintenance of the cold chain. However, cuts in energy supplies are recurrent in our capital. And this becomes a dilemma for Kinshasa consumers. What is the impact of the break in the cold chain on the quality of these frozen foods sold in the Kinshasa markets?

The objective of this research, based on isolation, identification and enumeration techniques and in accordance with the ISO standards specific to each germ, is to perform a microbiological analysis of the packaged, unpacked and cut chicken of the horse mackerel cardboard, exposed on stalls and local salted fish, imported and Congo River fish sold on some markets of Kinshasa, study the stakes of the safety by the method ISO-22000: 2005 HACCP and propose to the public authority the application of these principles of sanitation, to popularize them and to reinforce food hygiene measures in the large agglomerations of the country for the benefit of public health (accumulation of waste on the streets and sewer congestion) and to combat the toxicities Collective food infections (TIAC).

This study analysis shows that the frequency of contamination of micro-organisms, Proteus, top with 63\%, followed by Klebsiella 41\%, Escherichia coli 21\%, Citrobacters and Enterobacters $20 \%$ and Salmonella spp. And Shigella $8 \%$.

\section{INTRODUCTION}

La viande par sa grande valeur nutritive, reste un aliment très prisé. Elle est riche en nutriments, notamment en acides aminés essentiels qui font d'elle un aliment quasi irremplaçable. Elle présente également une bonne digestibilité, ce qui justifie, au moins en partie, le rapide développement dans le monde des industries des produits carnés et toutes les transactions commerciales y afférentes. Le plus gros problème en ce qui concerne la conservation de la viande, c'est le développement microbien. En effet, la viande constitue un excellent milieu de culture, un terrain favorable à la propagation et à la multiplication de germes pathogènes ou d'une charge microbienne importante, cela peut engendrer des problèmes sanitaires graves (Tubakila U.H et Sumbu M.E, 2011). Par définition on peut considérer que tout aliment après un contrôle alimentaire, est bon pour l'individu puisqu'il satisfait ses besoins (Cheftel J.C., et Cheftel H., 1977), Cheftel JC, Cheftel H et Besançon P., 1977, (Mokhadar, 2017). La définition communément admise du contrôle alimentaire par l'Organisation des Nations Unies pour l'alimentation et l'agriculture, le contrôle alimentaire est une activité de mise en application à caractère règlementaire, menée par des autorités nationales ou locales, visant à protéger les consommateurs et à garantir pour tous les aliments aux stades de la production, de la manutention, de l'entreposage, de la 
transformation et de la distribution, leur salubrité, leur pureté, et leur adaptation à la consommation humaine; leur conformité aux exigences de qualité et de sécurité sanitaire et enfin; l'honnêteté et l'exactitude de leur étiquetage conformément aux exigences de la loi. (EDES, www.coleacp.org/edes, consulté le 15 mai 2019). La République Démocratique du Congo est l'un des rares pays au monde à pouvoir regorger d'innombrables ressources humaines et naturelles susceptibles d'être rationnellement exploitées pour assurer sa croissance économique et surtout sa sécurité alimentaire tant dans les milieux urbains, périurbains que dans les zones rurales. Paradoxalement, la RD Congo importe des produits alimentaires et elle est enregistrée parmi les pays à faible revenu et à déficit vivrier en sigle Pays à Faible Revenu et à Deficit
Vivrier (PFRDV) (Kankonde et Tollens, 2001, FAO, 2011). Ces produits importés surtout ceux d'origine animale sont de médiocre qualité (Tollens, 2004, Kashala, 2008). Bien plus, depuis un certain temps, une baisse de leur consommation a été constaté suite à la diminution du pouvoir d'achat et au prix de ces produits importés (Tollens et Biloso, 2006). Le monde d'élevage tout comme celui de l'agroalimentaire sont agités au cours des dernières années par différentes crises: le cas d'intoxication alimentaire aux Etats-Unis avec la salmonellose et E. coli 015.H7 et l'épidémie en Europe d'encéphalopathie spongiforme bovine (ESB), grippe aviaire, grippe AH1N1. (Hanak et al, 2002). A émergé alors une remise en cause des élevages intensifs, des traitements hormonaux, antibiotiques.

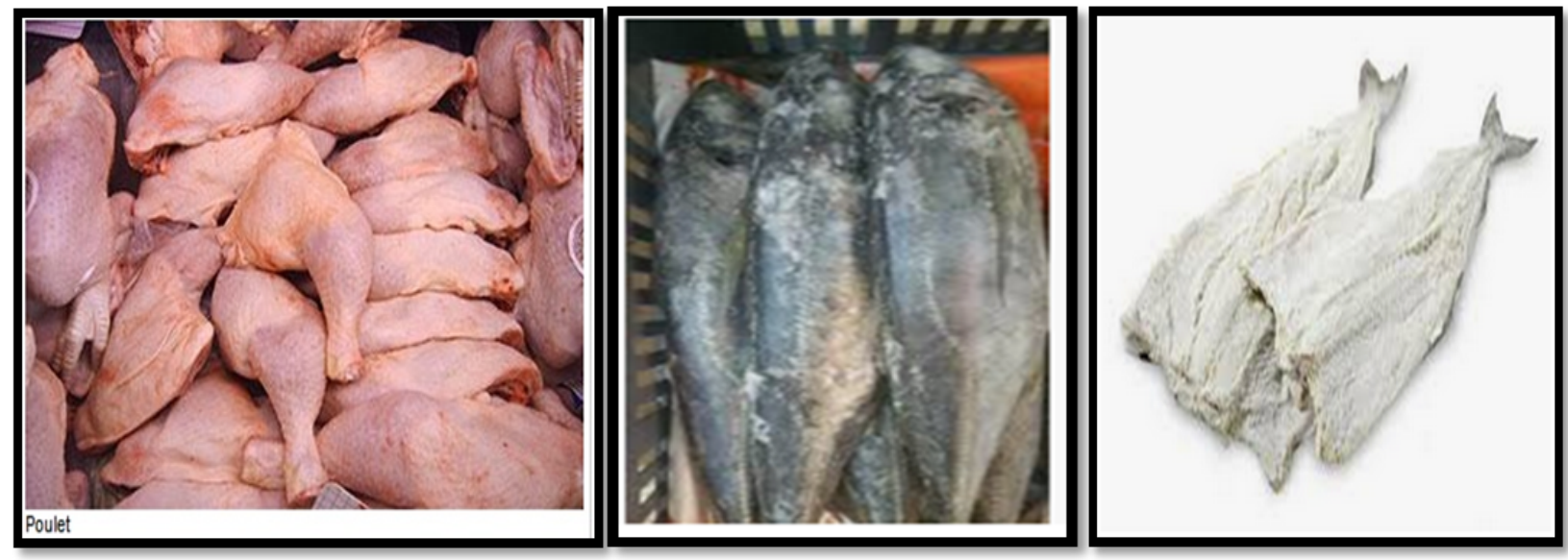

Photo 1 : Cuisses de poulet importé

Photo 2 : Poissons chinchards

Photo 3 : Poissons salés

Les importations en RD Congo de viande congelée du poulet surtout de chair en particulier, remonte en fait à l'indépendance du pays en 1960. C'est le gouvernement du premier ministre, Cyrille Adoula qui, le premier, ordonna ces importations. D'où la dénomination : «Ebembe ya Adoula », accolée déjà à l'époque à ces produits. Et presque tous les ménages consomment le «mpiodi» (chinchard), qui est ancré durablement dans les habitudes alimentaires du congolais démocratique. S'il y a des tares dans le chef de Congolais du moins ceux qui vivent dans les villes, à en croire (Seke, 2015), et qui participent ou contribuent au sous-développement ou à l'absence du progrès au pays, c'est bien cette tradition malheureuse de nos compatriotes de vouloir consommer extérieur ou des biens importés. Paradoxalement, elle importe chaque année plus de deux millions de tonnes de vivre via ses ports de Matadi et de Boma au Kongo Central et aux voies frontalières de 
Kasumbalesa dans le Haut-Katanga mais avec plus de 200.000 tonnes de poisson congelé. Pourtant, le fleuve Congo, les lacs et les rivières en RD Congo constituent des réserves halieutiques inestimables (Goossens et al., 1994; Goossens, 1996; Kankonde et Tollens, 2002), 80.000 tonnes de poulet et abats congelés, 185.000 tonnes de viandes de bœuf et de porc (FAO, 2004; FAO, 2006; Horman, 2006; http:/SADAOC.bf : Sécurité alimentaire durable en Afrique de l'ouest et en Afrique centrale; Ndonda, 2009). A en croire Seke (www.KongoTimes.info, 2015, consulté le 30 juin 2019), la RD Congo compte parmi les pays qui regorgent la plus grande réserve d'eau douce en Afrique et même au monde. Selon des experts, le lac Tanganyika est aligné comme le cours d'eau le plus poissonneux au monde. Où les poissons meurent de vieillesse. Mais qu'estce qui fait que les Congolais et principalement le Kinois et les populations de l'Ouest du pays s'acharnent à consommer le chinchard communément appelé « Mpiodi ». Des poissons importés du Danemark, du Portugal, de la Namibie ou des pays lointains d'autres continents. Ces poissons qu'on conditionne avec des produits chimiques et par le froid, le poisson «Mpiodi» souvent atteint le consommateur 2 à 4 mois après la pêche. Souvent ils sont dépourvus de toutes les vitamines et des vertus nourrissantes qui peuvent profiter à la santé humaine. Il se pose un problème de la sécurité alimentaire qui définit une situation aux niveaux individuel, familial, national, régional et mondial lorsque tous les êtres humains ont, à tout moment, un accès physique et économique à une nourriture suffisante, saine et nutritive leur permettant de satisfaire leurs besoins énergétiques et leurs préférences alimentaires pour mener une vie saine et active (FAO, 1996). Assurer la sécurité sanitaire des aliments d'origine animale nécessite d'agir à tous les stades de la chaîne alimentaire, de la production au niveau de la ferme jusqu'à la consommation par l'homme. De multiples risques sanitaires surviennent avant l'abattage des animaux, ou durant la phase de transformation. Ces risques peuvent être réduits ou prévenus par la politique de prévention et les bonnes pratiques préconisées par l'Organisation mondiale de la santé animale (OIE, 2015), (SADC, 2011). Le développement d'un pays est tributaire de plusieurs facteurs dont le plus important est incontestablement l'homme. L'état sanitaire de ce dernier est une nécessité absolue, car un morbide ou un malade est un être incapable d'exécuter un quelconque travail productif. Il va sans dire que la sauvegarde de la santé publique passe par une planification efficace et épidémio-surveillance adéquate. L'environnement kinois est réputé pour son insalubrité qui n'épargne pas les marchés et les endroits où sont commercialisés et consommés les aliments comme le poulet, le poisson. D'aucun n'ignore cette réalité. Pour prendre le cas des poulets et des poissons, ceux-ci sont étalés sans être protégés et lorsqu'ils le sont avec quel type d'emballage? Nous trouvons des poubelles non loin des étalages de vente dans les marchés et ceux qui sont étalés le long des routes et à même le sol, il y a tous ces échappements de la fumée des véhicules produisant de $\mathrm{CO}_{2}$. Ils sont transportés parfois dans des conditions douteuses: à bord de pousse - pousses, charrettes qui, en d'autres occasions, servent au transport de produits non alimentaires ne requérant pas de mesures d'hygiène particulières (Umba, 2002). Les coupures intempestives d'électricité ne favorisent-elles pas la détérioration de la qualité de ces poulets, poissons? Les vendeurs ou les acheteurs manipulent l'argent et touchent le poulet ou le poisson. Il y a une incidence sanitaire puisqu'il devient un facteur de propagation des germes pathogènes (Luamba et al., 2002). En RD Congo, peu d'études ont porté sur la relation entre l'ingestion des aliments et l'apparition de certains types de maladies (TIAC) (Masimango, 2014). Par ailleurs, la fréquence élevée des cancers primitifs du foie (C.P.F) observés dans les hôpitaux du pays ne serait-elle pas à mettre en rapport avec la consommation d'aliments contaminés par les aflatoxines? (Masimango et 
Kalengayi, 1983) . La question importante qui se pose est celle de savoir si les aliments importés, vendus et consommés en République Démocratique du Congo tels que les poulets et les poissons chinchards communément appelés «mpiodi » ainsi que les poissons salés ou les poissons $\mathrm{du}$ fleuve produits localement répondent à des normes microbiologiques du point de vue sécurité alimentaire ou selon le codex alimentarius? Quels types de microorganismes contiennent ces produits carnés tenant compte des conditions générales environnementales dans lesquelles ils sont vendus? Ils sont exposés à côté des immondices et aux intempéries du matin au soir (soleil et quand il pleut toutes eaux qui stagnent et la poussière...); sont vendus à proximité d'autres denrées : légumes, fruits divers, pains et pire, le long des principaux axes routiers où les véhicules circulent dégageant de la fumée...A cette question importante et celle intéressante s'ajoute celle de savoir l'incidence de la rupture de la chaine de froid car pour assurer l'intégrité de la qualité de ces poissons et ces poulets, la méthode utilisée est la congélation. En effet, le froid ralentit ou tout stoppe le développement des micro-organismes. Or, il y a un déficit énergétique avec des coupures intempestives de la fourniture électrique ou le phénomène de délestage au quotidien. Les coupures atteignent plusieurs heures. En fonction de la durée de la coupure d'électricité, la rupture de la chaîne de froid pourraient engendrer une mauvaise conservation de ces surgelés qui peuvent entraîner la prolifération des germes microbiens qui sont à l'origine des toxi-infections alimentaires. Ces bactéries peuvent être des

\section{MATERIEL ET METHODES}

\subsection{Matériels animal}

3.1.1 Échantillons de poulet et de poisson chinchard et salé : La période de notre étude s'est échelonnée du 10 janvier au 15 juin, 2019. Au total, 60 échantillons ont été récoltés pour analyse bactériologique. Les échantillons ont été analysés au laboratoire de l'INRB (Institut National de Recherche Biomédical) de salmonelles, des staphylocoques ou Listeria monocytogenes, dont l'ingestion peut occasionner des troubles gastriques (diarrhée), la fièvre ou aller jusqu'à la mort (Bakary, 2015). La sécurité alimentaire et la sécurité sanitaire à Kinshasa est un défi majeur! Du point de vue santé publique, existe-il des normes microbiologiques sur lesquelles la Police sanitaire et l'inspection des viandes de boucherie se basent pour déclarer les denrées hors normes; lesquelles peuvent occasionner des maladies zoonotiques réputées contagieuses. Par ailleurs est-il vrai de ces rumeurs qui courent dans toute la République Démocratique du Congo de l'utilisation du formol dans la conservation de la viande et le poisson importés. Cette solution chimique utilisée pour embaumer les personnes décédées? (Lemba, 2012). Il est un fait à en croire l'OMS, dans son document sur la sécurité sanitaire des aliments et santé AFR/ RC57/4 du 30 août 2007, la charge des maladies d'origine dans la Région Africaine est difficile à évaluer, mais les contaminants microbiens et chimiques des aliments constituent une source de préoccupation qu'il convient d'apaiser. Garantir la sécurité sanitaire des aliments est une responsabilité collective qui exige une vision commune de la part de toutes les parties prenantes. Telle est la préoccupation qui nous a poussée à commencer une étude sur le codex alimentarius en nous référant au système de HACCP (ISO$22000: 2005)$ et le vulgariser et en sensibilisant l'Etat congolais en faisant le contrôle de la qualité comme une priorité de la santé publique.

Kinshasa. Les échantillons de poulet et de poisson constituant les matériels de base, ont été prélevés auprès de plusieurs vendeurs à des points vente situés à huit endroits différents, à de moments différents de la journée et placés ensuite dans les emballages stériles dans les meilleures conditions d'asepsie. Pour permettre la représentativité de l'ensemble de la ville de 
Kinshasa, nous avons sélectionné huit sites pour la récolte des échantillons. Les critères de sélection étaient basés sur la répartition géographique de la ville, la concentration élevée de la population, les grands centres de ravitaillement (parkings de camions en provenance de l'intérieur du pays: Kongo Central, les provinces du Kwango, Kwilu et Maindombe et par voie fluviale pour l'ex province de l'Equateur.

\subsection{Matériels de prélèvement \\ - Gants \\ Sachets d'emballage \\ Bac isotherme \\ Accumulateur de froid \\ Marqueur noir \\ Etiquettes autocollant}

\subsection{Matériels pour la biosécurité}

- Gants en latex stériles

- Eau de Javel à 10\%

\subsection{Milieux de culture}

\subsubsection{Milieux d'isolement}

- Gélose Hektoen : ce milieu est sélectif pour l'isolement de Salmonella et Shigella. Toutefois d'autres germes pathogènes notamment les Vibrio peuvent également pousser

- Gélose Thiosulfate bile saccharose (TCBS) : est un milieu sélectif pour le Vibrio cholerae

Gélose Mannitol Salft Agar (MSA) : ce milieu est sélectif pour le Staphylococcus aureus - Gélose nutritive: est une gélose ordinaire pour tout germe

\subsubsection{Milieu d'enrichissement}

Bouillon Sélénite : c'est un milieu pour l'enrichissement de Salmonella

Bouiillon Eau peptonée alcalin (EPA) : c'est un bouillon réservé à l'enrichissement des Vibrio cholerae

- Bouillon Eau peptonée Tamponnée (EP) : c'est un milieu non inhibiteur

\subsubsection{Milieu d'identification}

Gélose Kligler iron Agar: pour la recherche de quatre caractères biochimiques suivants: la fermentation lactose et glucose.
Production de gaz dans le glucose et noircissement par production de $\mathrm{H}_{2} \mathrm{~S}$

- Gélose Mannitol Mobilité : pour la recherche de la fermentation du sucre mannitol et la mobilité de la bactérie

Citrate de Simmons: pour déterminer l'utilisation du citrate par la bactérie

\subsubsection{Milieu de dénombrement}

Gélose Bromocrésol Pourpre (BCP): milieu pour le dénomrement de la flore globale

3.6 Réactifs utilisés sont : d'indole)

- Disque d'oxydase

- Disque d'orthophényl galactosidase (ONPG)

- $\quad$ Kit réactif pour la coloration gram

3.7 Antisérums : Nous avons disposé de quelques antisérums

- $\quad$ Antisérums salmonella 0 (A-S)

- $\quad$ Antisérums polyvibrio 01

- $\quad$ Antisérums Shigella flexineri

- $\quad$ Antisérums Shigella boydii

$4 \quad$ Méthodes de travail adoptées

4.1 Prélèvement, conservation et transport des échantillons: L'étape du prélèvement était très importante pour notre étude, elle a été réalisée en 2 phases: la première phase était réservée à la prospection et sélection des sites de prélèvement qui devaient en faire partie de notre étude. Cette prospection a été réalisée une semaine avant le début de prélèvement des échantillons. Les responsables des dépôts ainsi que certains vendeurs ont été contactés soit le jour de la prospection, soit le jour même du prélèvement. La deuxième phase était consacrée au prélèvement des échantillons pour analyse.

4.2 Mode opératoire : Les matériels de prélèvement étaient préparés la veille. Les sachets plastiques étaient stérilisés aux UV, tandis que d'autres matériels ont été lavés et apprêtés pour être utilisés le lendemain. Les prélèvements s'effectuaient à des heures différentes de la journée et chaque prélèvement se réalisait dans les conditions d'asepsie. A chaque prélèvement: les mains étaient 
désinfectées avec de l'eau de Javel à 10\% avant de porter les gants en latex stériles. Après avoir sélectionné l'échantillon, il a été introduit dans l'emballage stérile, étiqueté et introduit dans le bac isotherme contenant des accumulateurs de froid $\left(+4^{\circ} \mathrm{C}\right.$ basse température). Au niveau de chaque site, les différentes catégories d'échantillons ont été prélevées puis acheminés rapidement au laboratoire pour analyse. Le transport entre le lieu de prélèvement et le laboratoire n'excédait pas en moyenne une heure.

\subsubsection{Traitement bactériologique}

a) Dénombrement de la flore mésophyle : Le dénombrement de la flore aérobie mésophyle s'effectuait par ensemencement sur le milieu gélosé de numération Bromocrésol pourpre $(\mathrm{BCP})$ en boîte de pétrie à partir d'un millilitre de dilution dans l'eau peptonée tamponnée.

b) Examen direct: Examen direct après coloration

\section{- $\quad$ Principe :La coloration de Gram est une} coloration différentielle qui permet de distinguer deux types de bactéries: Gram positif et Gram négatif. Les bactéries Gram positif forment un complexe indissociable avec le violet de gentiane, après l'action de l'alcool acétone, elles apparaissent bleu-violettes, tandis que les bactéries Gram négatif sont colorées en rouge-rose.

\section{RESULTATS ET INTERPRÉTATION}

Les poissons sont extrêmement périssables. Cela est dû aux phénomènes d'altération postmortem ayant pour origine les réactions autolytiques et les changements bactériologiques (Diagne, 1995; Clucas, 1986). Les pertes commencent dès que celui-ci meurt ou capturé (Thian, 1993). Sous les tropiques o la température est très élevée $\left(25\right.$ à $\left.30^{\circ} \mathrm{C}\right)$, le poisson s'altère au bout de 12 à 20 heures selon les espèces (Clucas, 1986). Les résultats des analyses microbiologiques sont consignés dans cinq tableaux. L'infestation du poulet, et du poisson vendus à Kinshasa par les microorganismes paraît effroyable. A différents points de la chaîne de distribution, la contamination est importante. D'autre part, le poisson et le poulet sont des aliments les plus consommés par la population kinoise; même le plus démuni des kinois, incapable de se procurer un filet de viande quotidien, ne manquera pas de manger ne fut-ce qu'un morceau de poulet, ou de poisson, même s'il n'est pas capable d'acheter une pièce entière.

Tableau 1 : Répartition des échantillons par nature

\begin{tabular}{|l|l|}
\hline Nature & Nombre en $\%$ \\
\hline Poulet emballé & $5(8,3)$ \\
\hline Poulet déballé & $5(8,3)$ \\
\hline Poulet découpé & $5(8,3)$ \\
\hline Poisson chinchard sorti du carton & $10(16,6)$ \\
\hline Poisson chinchard exposé & $10(16,6)$ \\
\hline Poisson salé local & $8(13,3)$ \\
\hline Poisson salé importé & $7(11,6)$ \\
\hline Poisson du fleuve Congo & $10(16,6)$ \\
\hline \multicolumn{1}{|c|}{ Total } & 60 échantillons \\
\hline
\end{tabular}

5.1 Interprétation : Au vu de ce tableau 1, nous constatons que sur 60 échantillons examinés, les différentes catégories de poissons ont constitué la proportion la plus de nos échantillons, soit $16,6 \%$ pour le poisson chinchard sorti du carton, $16,6 \%$ pour le poisson chinchard exposé et $16,6 \%$ pour le poisson du fleuve, le poisson salé local et le 
poisson salé importé occupent respectivement 13 et 11,6\%. Par contre les poulets examinés ne représentaient qu'une proportion assez faible des échantillons soit $8,3 \%$ pour le poulet emballé, $8,3 \%$ pour le poulet déballé et $8,3 \%$ pour le poulet découpé.

Tableau 2 : Répartition des prélèvements par sites

\begin{tabular}{|c|c|}
\hline Sites & Nombre d'échantillons $\%$ \\
\hline Marché central & $6(10)$ \\
\hline Marché Gambela & $14(23,3)$ \\
\hline Marché Pascal & $6(10)$ \\
\hline Marché Kingasani ya suka & $8(13,3)$ \\
\hline Marché Rond-point Ngaba & $10(16,6)$ \\
\hline Marché Libongo / Beach & $1(1,6)$ \\
\hline Marché UPN & $8(13,3)$ \\
\hline Marché Kinkole & $10(16,6)$ \\
\hline Total & 60 échantillons \\
\hline
\end{tabular}

5.2 Interprétation: Nous avons dressé ce tableau reprenant les nombres d'échantillons récoltés par marché sélectionné. Ilo ressort de ce tableau que 8 marchés de Kinshasa ont été sélectionnés en tenant de l'ouverture de vendeurs et l'accès des marchés. Il s'agit des marchés : Central, Gambela, Pascal, Kingasani ya suka, Rond-Point Ngaba, UPN, Kinkole et
Libongo/Beach. Au regard de ce tableau, sur 60 échantillons examinés, 14 soit $(23,3 \%)$ provenaient du Marché Gambela, suivis des marchés Kinkole et Rond-point Ngaba avec 10 échantillons chacun, soit 16,6\%). Nous avons constaté qu'un échantillon $(1,6 \%)$ provenait de Libongo / Beach Ngobila étant donné qu'il s'agissait seulement du poisson salé local.

Tableau 3 : Fréquence de contaminations des échantillons souillés

\begin{tabular}{|l|c|}
\hline Types des micro-organismes rencontrés & Cas isolés \\
\hline Proteus & 38 \\
\hline Escherichia coli & 13 \\
\hline Citrobacter & 12 \\
\hline Klebsiella & 25 \\
\hline Enterobacter & 12 \\
\hline Bacilles gram(-) non enterobactéries & 6 \\
\hline Salmonella & 5 \\
\hline Shigella & 5 \\
\hline Morganella & 2 \\
\hline Vibrio non cholerae & 3 \\
\hline Pseudomonas & 1 \\
\hline Providencia & 4 \\
\hline
\end{tabular}

5.3 Interprétation: Nous avons classifié dans le tableau 3, la fréquence de contaminations par différents germes des échantillons, examinés dans la les mêmes conditions de culture bactériologique: le
Proteus présente la fréquence la plus élevée car on le retrouve dans 38 échantillons, Klebsiella retrouvé dans 25 échantillons est le microorganisme les plus fréquent après le Proteus. Nous constatons également qu'un certain 
nombre d'échantillons sont souillés par les germes de contamination fécale tels que: Salmonella, Shigella et Vibrion non Cholerae. D'autres germes ont été également mis en évidence notamment, l'Enterobacter: 12 échantillons sont souillés: Morganella: 2 échantillons souillés et aussi Escherichia coli avec 13 échantillons contaminés, Providencia a été retrouvé dans 4 échantillons et d'1 échantillon de Pseudomonas.

Tableau 4 : Répartition des germes pathogènes par type d'échantillon

\begin{tabular}{|l|l|l|l|l|l|l|l|l|}
\hline $\begin{array}{l}\text { Types } \\
\text { d'échantillons }\end{array}$ & $\begin{array}{l}\text { Poulet } \\
\text { emballé }\end{array}$ & $\begin{array}{l}\text { Poulet } \\
\text { déballée }\end{array}$ & $\begin{array}{l}\text { Poulet } \\
\text { découpé }\end{array}$ & $\begin{array}{l}\text { Poisson } \\
\text { chinchard } \\
\text { sorti du } \\
\text { carton }\end{array}$ & $\begin{array}{l}\text { Poisson } \\
\text { chinchard } \\
\text { exposé }\end{array}$ & $\begin{array}{l}\text { Poisson } \\
\text { salé } \\
\text { local }\end{array}$ & $\begin{array}{l}\text { Poisson } \\
\text { salé } \\
\text { importé }\end{array}$ & $\begin{array}{l}\text { Poisson } \\
\text { du } \\
\text { fleuve }\end{array}$ \\
\hline Germes & & & & & & & & \\
\hline Salmonella & 1 & 1 & Pas & 2 & $*$ & 1 & $*$ & $*$ \\
\hline Shigella & 2 & 1 & de & 1 & 1 & $*$ & $*$ & $*$ \\
\hline Esh. Coli & 3 & 1 & germes & 2 & 3 & 3 & 3 & $*$ \\
\hline $\begin{array}{l}\text { Vibrio non } \\
\text { cholerae }\end{array}$ & 2 & $*$ & pathogènes & 1 & $*$ & $*$ & $*$ & $*$ \\
\hline Pseudo monas & $*$ & $*$ & isolés & 1 & $*$ & $*$ & $*$ & $*$ \\
\hline
\end{tabular}

5.4 Interprétation : Nous avons réparti les différents échantillons examinés en fonction des germes isolés. Ainsi, nous constatons que :

- Sur 5 échantillons de poulets emballés :

- 1 est souillé par Salmonella

- 2 sont souillés par Shigella

- $\quad 3$ sont souillés par Escherichia coli

- $\quad 2$ sont souillés par Vibrio non cholerae

- $\quad$ Sur 5 échantillons de poulet déballés

- 1 est souillé par Salmonella

- 1 est souillé par Shigella

- $\quad 1$ est souillé par Escherichia coli
Il est à noter que, les poissons chinchard sortis du carton sont tous contaminés par les différents germes de pollution. Aucun poulet découpé examiné n'était contaminé. Les germes tels que Salmonella et Vibrio non cholerae n'ont pas été mis en évidence chez le poisson chinchard exposé, le poisson local, et le poisson salé importé. De même tous les échantillons des catégories examinés, à l'exception du poulet découpé, contenaient Escherichia coli, germe de contamination fécale.

Tableau 5 : Répartition des échantillons souillés par marché

\begin{tabular}{|l|l|l|l|l|l|l|}
\hline & $\begin{array}{l}\text { Marché } \\
\text { central }\end{array}$ & $\begin{array}{l}\text { Marché } \\
\text { Kingasani ya } \\
\text { suka, Pascal }\end{array}$ & $\begin{array}{l}\text { Marché } \\
\text { Rond-point } \\
\text { Ngaba }\end{array}$ & $\begin{array}{l}\text { Marché } \\
\text { Gambela }\end{array}$ & $\begin{array}{l}\text { Marché } \\
\text { Kinkole }\end{array}$ & $\begin{array}{l}\text { Marché } \\
\text { UPN }\end{array}$ \\
\hline 1. Proteus & 2 & 10 & 6 & 9 & 11 & 10 \\
\hline 2. Esch. Coli & $*$ & 4 & 3 & 4 & 1 & 2 \\
\hline 3. Citrobacter & 1 & 5 & 1 & 2 & 3 & $*$ \\
\hline 4. Klebsiella & 1 & 6 & 7 & 6 & 4 & 4 \\
\hline 5. Enterobact. & 4 & 4 & $*$ & 3 & $*$ & $*$ \\
\hline $\begin{array}{l}\text { 6. Bacille gram (-) } \\
\text { non enterobact }\end{array}$ & $*$ & 1 & 2 & 1 & $*$ & 2 \\
\hline 7. Salmonella & $*$ & $*$ & 2 & 3 & $*$ & 2 \\
\hline 8. Shigella & $*$ & $*$ & 1 & 2 & $*$ & $*$ \\
\hline 9. Morganella & $*$ & $*$ & 2 & $*$ & $*$ & $*$ \\
\hline
\end{tabular}




\begin{tabular}{|l|l|l|l|l|l|l|}
\hline $\begin{array}{l}\text { 10. Vibrio non } \\
\text { cholerae }\end{array}$ & $*$ & $*$ & $*$ & 3 & $*$ & $*$ \\
\hline 11. Pseudo monas & $*$ & $*$ & $*$ & 1 & $*$ & $*$ \\
\hline 12. Providencia & $*$ & $*$ & $*$ & $*$ & 4 & $*$ \\
\hline
\end{tabular}

5.5 Interprétation: Au regard de ce tableau 5, nous constatons que les échantillons qui provenaient des marchés Gambela et Rondpoint Ngaba sont plus contaminés et par plusieurs germes différents. Trois échantillons contaminés par Salmonella provenaient du marché Gambela, deux provenaient du marché Rond-point Ngaba. De même deux échantillons et un autre souillés par Shigella provenaient respectivement du marché Gambela et du Rond-point Ngaba. Deux autres échantillons

\section{DISCUSSION ET CONCLUSION}

La présente étude s'intéresse à la santé publique et au respect de la législation. En effet, pour qu'un aliment puisse remplir son rôle de couvrir les dépenses énergétiques de l'organisme qui est au travail ou en croissance, il convient qu'il ne soit pas ni toxique ni malsain, c'est-à-dire qu'il ne contient aucun élément, de nature chimique (antiseptique, produit ajouté) qu'il soit indemne des germes pathogènes et que son éventuelle teneur en germes banaux soit comprise dans les limites tolérables avec bonne qualité (Bonnard, 2001 et Faiza, 2013. La FAO, signalait dans une étude publiée à Kinshasa, que plus de $80 \%$ des aliments vendus dans les lieux publics, via le secteur informel, étaient contaminés principalement par le Bacillus cereus, Salmonella, Shigella, Escherichia coli et même Vibrio cholerae, (Horman, 2006). Et nos résultats se complètent avec ceux d'UNAGRICO et la FAO qui signalaient que $80 \%$ des aliments vendus dans les lieux publics, via le secteur informel, étaient contaminés, principalement par le Bacillus aureus, Salmonella, Shigella, Escherichia coli et même Vibrio cholerae. Les surgelés comptent parmi les aliments consommés dans les grandes villes de la $\mathrm{RD}$ Congo. Cependant, une bonne partie de ces produits subit une rupture de chaîne de froid telle qu'elle détériore et devient nuisible à la souillés par Shigella étaient récoltés au marché de l'UPN/ Université Pédagogique Nationale. Aussi, trois échantillons contaminés par Vibrio non cholerae provenaient du marché Gambela. Par contre nous constatons que les germes pathogènes d'origine fécale tels que Salmonella, Shigella, Vibrio non cholerae n'ont pas été isolés sur les échantillons récoltés aux marchés Central, Kingasani ya suka, Kinkole, Pascal et Libongo /Beach Ngobila.

santé des consommateurs (Kuhinia, 2013 et Borges et al, 2013). Il serait trop téméraire et même risqué à ce niveau de se livrer à une réflexion fut-elle pertinente dans une discipline comme le HAACP, un sigle anglais qui signifiant: "Hazard Analysis Critical Control Point». L'équivalent français est: Analyse des Dangers et Contrôle des Points Critiques pour leur Maitrise ». C'est une méthode qui analyse des risques et la maitrise des points critiques du point de vue sécurité alimentaire et santé publique au regard des importations des produits congelés qui entrent à partir du port de Matadi ou de la consommation de viande e volaille et du poisson à Kinshasa. Et l'intervention de l'autorité politique s'impose car la majorité des vendeuses affirment ne pas être au courant de cette réalité. L'Etat doit veiller à la sécurité des produits à consommer quelle que soit sa destination depuis sa fabrication jusqu'à son utilisation. En République Démocratique du Congo, la mission de veiller à la sécurité des produits à consommer quelque soit sa destination depuis sa fabrication jusqu'à la dernière utilisation a été confiée à l'OCC (Office Congolais de Contrôle), une institution régit par l'ordonnance-loi no 74/013 du 10 juillet 1974 . L'objectif premier de cette étude était de 
répondre à la question de savoir si les aliments importés, vendus et consommés è Kinshasa en RD Congo tels que les poulets, les poissons chinchard, les poissons produits localement et les poissons salés répondaient à des normes microbiologiques et cela en tenant compte de l'environnement kinois et d'appuyer les efforts de l'OCC. Le deuxième objectif était de réfléchir du fait que les surgelés qui comptent parmi les aliments les plus consommés dans les grandes villes mais mal conservé sur les marchés était un problème de santé pour les

\section{BIBLIOGRAPHIE}

Bakary, A.B. (2015) Impact de la rupture de la chaine de froid sur la qualité bactériologique de Scombers scombrus (maquereau commun), Trachurus (chinchard) dans le sud Bénin. Mémoire de Master, École polytechnique d'Abomey-Calavi-Faculté des sciences agronomiques et Faculté des Sciences et techniques- Université d'AbomeyCalavi, inédit, $54 \mathrm{p}$.

Berryhill, J., Bourgery T. et Hanson A. (2018) "Blockchains Unchained: Blockchain Technology and its Use in the Public Sector». OECD, Working Papers on Public Governance, $\mathrm{n}^{\circ}$ 28, OECD Publishing, Paris. Consulté en ligne http://www.fao-whocodexalimentarius/codextexts/guidelines/fr/

Bonnard, R. (2001) Le risque biologique et la méthode d'évaluation du risque. Rapport final-Ministère de l'Aménagement du Territoire et de l'Environnement. INERIS (Institut National de l'Environnement Industriel et des Risques), 15 novembre 2001, inédit, 79 p.

Borges, F., Burtin H, Cheruel A, Collu E, Dudognon E, Moureau C, Schmit C, Pace H, et Plessis M. (2014) Sécurité sanitaire des aliments. Université de Lorraine $54 \mathrm{p}$. consommateurs kinois? Et enfin le troisième objectif était de déterminer (numération et identification) les divers micro-organismes contaminant éventuellement ces aliments. 60 échantillons ont été prélevés dans 8 sites, à différents moments de la journée. Les analyses faites dans les conditions appropriées ont permis d'isoler quelques germes pathogènes à savoir Salmonella, Shigella, Escherichia coli et Vibrion non cholerae surtout dans le poulet emballé et dans le poisson chinchard sorti du carton.

Cheftel J.C et Cheftel H., (1977) Introduction à la biochimie et à la technologie des aliments. Volume 1, Technique et Documentation-Lavoisier, 11 rue Lavoisier- F75384 Paris Cedex 08, 381 $\mathrm{p}$.

Cheftel J.C.; Cheftel H.; et Besançon P., (1977) Introduction à la biochimie et à la technologie des aliments. Volume 2, Technique et Documentation-Lavoisier, 11 rue Lavoisier-F75384 Paris Cedex 08, $419 \mathrm{p}$.

Clucas, J. (1986) Manutention, conservation et transformation $\mathrm{du}$ poisson sous les tropiques, partie 2, Wageningen, PaysBas, CTA, 14 p.

Diagne, M.A. (1995) Contribution à la détermination de l'indice de fraicheur de quelques espèces de poissons tropicales. Th. Méd. Vét. Dakar, n²4, 75 p.

EDES, www.coleacp.org/edes consulté le 15 mai 2019

Encyclopédie Wikipédia, (2007) consulté le 15 mai 2019.

Faiza, E. (2013) Évaluation de la qualité microbiologique des eaux d'alimentation humaine. Université Sidi Mohamed Ben Abdellah-Faculté des Sciences et Techniques-FES, Rapport de stage 11 juin 2013, inédit, $33 \mathrm{p}$.

FAO (1996) Déclaration de Rome sur la sécurité alimentaire mondiale et Plan 
d'action du SMA. Sommet Mondial de l'alimenation 13-17 novembre, Rome.

FAO (2006) Food and Agriculture Organization, Statistical Yearbook 2005-2006. Vol.2/1 and 2/2, (FAO, Rome)

FAO (2011) Plan d'action pour la gestion des risques de catastrophe en République Démocratique du Congo, Division des opérations d'urgence et de la réhabilitation, Rome, $56 \mathrm{p}$.

FAO (2019) www.fao,org/republiqueDémocratqiue-congo/fr/

FAO Sécurité sanitaire des aliments consulté en ligne $\quad$ http://www.fao.org/food$\underline{\text { Safety } / \mathrm{fr} /}$

FAO/OMS Garantir la sécurité sanitaire et la qualité des aliments : Directives pour le renforcement des systèmes nationaux de contrôle alimentaire consulté en ligne http://www.fao.org/3/a-y8705f.pdf

FAOSTAT (2004) Agricultural production, crop primary database. Food and Agricultural Organization of the United Nations, Rome. http:/ / faostat.org/ faostat/

Goossens, F. (1996a) Commercialisation des vivres locaux en Afrique Subsaharienne, le secteur informel dans un perspectif dynamique. Une contribution au projet GCP/RAF/309, AGSM, FAO, novembre 1996, $58 \mathrm{p}$.

Goossens, F. (1996b) Rôle des systèmes d'alimentation dans la sécurité alimentaire de Kinshasa. Une contribution au projet GCP/RAF/309/ AGSM, FAO, mai 1996, 78 p.

Goossens, F., Minten B., et Tollens, E. (1994) Nourrir Kinshasa : l'approvisionnement local d'une métropole africaine. L'Harmattan, Paris, 397 p.

Hanak, E., Boutrif, E., Fabre, P. et Pineiro, M. (2002) La gestion de la sécurité des aliments dans les pays en développement. Actes de l'atelier international CIRAD-FAO, 11-13 décembre 2000. Montpellier, France.
CIRAD-FAO, Cederom du CIRAD, Montpellier-France.

Horman, D. (2006) Kinshasa et les importations avicoles- En finir avec la catastrophe sanitaire et économique. Cahiers d'Alternatives $\mathrm{n}^{\circ} 7$, GRESEA, $28 \mathrm{p}$.

http:/SADAOC.bf. : Sécurité alimentaire durable en Afrique de l'ouest et en Afrique centrale

https://fr.wikibooks.org/...microbiologie...alim ents $/ \mathrm{Qu} \% 27$ est-ce qu $\% 27 \mathrm{u}$

Huss, H.H. (1988) Le poisson frais : sa qualité et altération de qualité Rome, FAODANIDA, Collection FAO, 132 p.

Kankonde, M. et Tollens E., (2001) Sécurité alimentaire au CongoKinshasa/Production, Consommation et Survie. Éditions l'Harmattan 5-7, rue de l'École Polytechnique-75005 ParisFrance.

Kashala, K.J.C. (2008) La recherche agronomique et le développement durable de l'agriculture congolaise. Presses Universitaires de Lubumbashi, Éd. Salama Don-Bosco, Lubumbashi, $100 \mathrm{p}$.

Kuhinda, C. (2013) Les surgelés mal conservés sur les marchés de Kinshasa, problème de santé pour les consommateurs. Consulté en ligne www.speakjhr.net/news/les-produitscongelés-importés-font-le-regal-descongolais

Lemba, S.T. (2012) L'usage du formol dans les vivres frais- Un danger public! Consulté le 10 janvier 2019 en ligne https://www.lecongolais.cd/SANTE23 août 2012

Luamba, L.N, Daya, N., Bitumba B., Tabu M., et Nkunga M., (2002) Incidence sanitaire des signes monétaires en circulation à Kinshasa. Département de Biologie, IPN/Binza-Kinshasa.

Masimango, T.N. (2014) Microbiologie des aliments. Notes de cours, premier grade, Département de Chimie et Industries agricoles. Faculté des Sciences 
Agronomiques. Université de Kinshasa, inédit, $53 \mathrm{p}$.

Masimango, T.N. et Kalengayi, M. (1983) Aflatoxins in food and Foodstuffs in Zaïre, In Proc.Int.Symp.Myctoxins, pp 431-435, F.D.A. Rockville, Maryland.

Masimango, T.N., (2005) Questions spéciales des industries agroalimentaires. Aflatoxines, Faculté des Sciences Agronomiques, Inédit, Kinshasa, 40 p.

Mokhadar M., (2017) Contrôle de la qualité physic-chimique et microbiologique de la viande de poulet. Mémoire de MasterFaculté des Sciences de la Nature et de la vie et Sciences de la Terre et de l'Univers, Département de BiologieUniversité Abou Bakar Belkaid Tlemcen, inédit $69 \mathrm{p}$.

Ndonda, J.L. (2009) L'incidence des importations et aides alimentaires sur l'agriculture congolaise consulté en ligne https://www.memoireonline.com/a/fr Lart/Show consulté le 20 janvier 2019

Nzuzi Lelo N.F., (2008) Kinshasa-Ville et environnement, l'Harmattant 5-7 rue de l'École Polytechnique: 75005, Paris, $281 \mathrm{p}$.

OIE (2015) La sécurité sanitaire des aliments. Fiches repères,

SADC (2011) Directives régionales pour la règlementation de la sécurité sanitaire des aliments dans les états membres de la SADC. Projet de Sécurité Sanitaire des aliments- Renforcement des capacités du Contrôle des Résidus, FANR. 59 p.

Seke, J.P (2015) RDC, consommer congolais. Consulté en ligne www.Kongo'Times.info
Thian, A.A. (1993) Contribution à l'étude de la qualité microbiologique et chimique de poisson braisé-séché (ketiak) commercialisé sur le marché Dakarois. Th.Méd Vét. Dakar, n¹5, 85 p.

Tollens, E. (2004) Les défis : sécurité alimentaire et cultures de rente pour l'exportation. Principales orientations et avantages comparatifs de l'agriculture en RD Congo. Working paper 2004/86. Faculté des Sciences agronomiques et de la biologie appliquée- Katholeike Universiteit Leuven. 76 pages

Tollens, E. et Biloso, A. (2006) République Démocratique du Congo. Profil des marchés pour les évaluations d'urgence de la sécurité alimentaire- Katholieke Universiteit Leuven, 94 pages.

Tubakila U.H. et Sumbu, M.E (2011) Toxicité aminée dans la viande en putréfaction. Travail de fin de cycle- Faculté des Sciences Agronomiques, Université de Kinshasa. Inédit, 30 pages.

Umba, J. (2002) Contribution à l'étude microbiologique des aliments consommés en l'état à Kinshasa, cas du pain. Mémoire de DES, Faculté des Sciences Agronomiques, Université de Kinshasa, 74 p publié sous forme d'article au Journal of Animal \& Plant Sciences, 2018, vol. 38, issue 2 : 62446256 en ligne http://www.m.elewa.org/JAPS,ISSN20 71-7024.

www.congo-autrement.com/.../ville-provinede-kinshasa/rdc-la-population-de-laville-...

www.KongoTimes.info,2015 consulté le 30 juin 2019 